\title{
Population-Based Study of Pseudoprogression after Chemoradiotherapy in GBM
}

\author{
Gloria B. Roldán, James N. Scott, John B. McIntyre, Marisa Dharmawardene, \\ Paula A. de Robles, Anthony M. Magliocco, Elizabeth S. Y. Yan, Ian F. Parney, \\ Peter A. Forsyth, J. Gregory Cairncross, Mark G. Hamilton, Jacob C. Easaw
}

\begin{abstract}
Introduction: Chemoradiotherapy followed by monthly temozolomide (TMZ) is the standard of care for patients with glioblastoma multiforme (GBM). Case reports have identified GBM patients who experienced transient radiological deterioration after concurrent chemoradiotherapy which stabilized or resolved after additional cycles of adjuvant TMZ, a phenomenon known as radiographic pseudoprogression. Little is known about the natural history of radiographic pseudoprogression. Methods: We retrospectively evaluated the incidence of radiographic pseudoprogression in a population-based cohort of GBM patients and determined its relationship with outcome and MGMT promoter methylation status. Results: Out of 43 evaluable patients, 25 (58\%) exhibited radiographic progression on the first MRI after concurrent treatment. Twenty of these went on to receive adjuvant TMZ, and subsequent investigation demonstrated radiographic pseudoprogression in 10 cases (50\%). Median survival (MS) was better in patients with pseudoprogression (MS 14.5 months) compared to those with true radiologic progression (MS 9.1 months, p=0.025). The MS of patients with pseudoprogression was similar to those who stabilized/responded during concurrent treatment ( $\mathrm{p}=0.31)$. Neither the extent of the initial resection nor dexamethasone dosing was associated with pseudoprogression. Conclusions: These data suggest that physicians should continue adjuvant TMZ in GBM patients when early MRI scans show evidence of progression following concurrent chemoradiotherapy, as up to $50 \%$ of these patients will experience radiologic stability or improvement in subsequent treatment cycles.
\end{abstract}

RÉSUMÉ: Étude de population sur la pseudoprogression après la chimioradiothérapie dans le glioblastome multiforme. Introduction : Le traitement standard du glioblastome multiforme (GBM) est la chimioradiothérapie suivie de l'administration mensuelle de témozolomide (TMZ). Certains cas de patients atteints de GBM, qui ont présenté une détérioration radiologique transitoire après la chimioradiothérapie qui s'est stabilisée ou s'est résorbée après des cycles additionnels de TMZ, un phénomène connu sous le nom de pseudoprogression radiologique, ont été rapportés. Méthodes : Nous avons évalué rétrospectivement l'incidence de la pseudoprogression radiologique chez une cohorte de patients atteints de GBM tirée de la population et nous avons déterminé sa relation avec le résultat du traitement et avec l'état de méthylation du promoteur MGMT. Résultats : Vingt-cinq des 43 patients évaluables $(58 \%)$ présentaient une progression radiologique à la première IRM après le traitement. Vingt d'entre eux ont reçu du TMZ comme thérapie adjuvante et une évaluation subséquente a montré une pseudoprogression radiologique chez 10 patients (50\%). La survie médiane (SM) était meilleure chez les patients qui présentaient une pseudoprogression (SM de 14,5 mois) comparée à celle des patients qui présentaient une véritable progression radiologique (SM 9,1 mois, $\mathrm{p}=0,025)$. La SM des patients qui présentaient une pseudoprogression était semblable à celle des patients qui se sont stabilisés/ont répondu au traitement concomitant $(\mathrm{p}=0,31)$. Ni l'étendue de la résection initiale ni le dosage de la dexaméthasone n'était associé à la pseudoprogression. Conclusions : Ces données sont en faveur d'une poursuite du traitement adjuvant par le TMZ dans le GBM chez les patients dont les scans IRM montrent une progression suite à la chimioradiothérapie parce que jusqu'à $50 \%$ de ces patients présenteront une stabilisation radiologique ou une amélioration lors des cycles de traitement subséquents.

Can. J. Neurol. Sci. 2009; 36:617-622

Patients with glioblastoma (GBM), the most common primary brain tumor in adults, have a median survival of about one year. In 2005, Stupp et $\mathrm{al}^{1}$ published the results of a randomized controlled clinical trial in GBM demonstrating a significant survival benefit by adding temozolomide (TMZ) to radiation. In this study, patients with newly diagnosed GBM were treated with low dose temozolomide daily during radiation therapy (termed "concurrent treatment") followed by six monthly cycles of TMZ administered five davs out of every 28
From the Department of Oncology (GBR, JBM, MD, PAD, AMM, PAF, JCE), Tom Baker Cancer Centre, Alberta Cancer Board; Department of Clinical Neurosciences (GBR, PAD, PAF, JGC, MGH), Department of Diagnostic Imaging (JNS), Department of Pathology \& Lab Medicine (JBM, AMM), University of Calgary; Clark Smith Integrated Brain Tumor Research Centre (PAF, JGC, MGH, JCE), Heritage Medical Research Building, Calgary, AB, Canada; Department of Radiation Oncology (ESYY), Department of Neurologic Surgery (IFP), Mayo Clinic, Rochester MN, USA. Received January 5, 2009. Final Revisions Submitted April 29, 2009. Correspondence to: Jacob Easaw, Room 111G, Tom Baker Cancer Centre, 1331 29th Street NW, Calgary, Alberta, T2N 4N2, Canada. 
days. With this approach, survival at two years improved from $10 \%$ to $26 \%{ }^{1}$. Hegi et $\mathrm{al}^{2}$ observed that $\mathrm{O}^{6}$-methylguanine-DNA methyltransferase (MGMT) promoter methylation status was associated with survival benefit from this therapy. The two-year survival rate in patients with tumors harboring methylated MGMT promoters was $46 \%$, compared to $13.8 \%$ in patients with unmethylated tumors. Currently, concurrent chemoradiotherapy followed by monthly temozolomide is the standard of care for patients with GBM.

Some of the earliest reports describing the use of TMZ and radiation for high grade gliomas reported transient clinical or radiological deterioration which later resolved in patients who continued adjuvant TMZ. Distinguishing treatment-induced imaging changes, also known as radiographic pseudoprogression $^{3-7}$, from true progressive disease can be especially difficult in the period immediately following completion of radiotherapy (RT). Taal et $\mathrm{al}^{5}$ concluded that nearly $50 \%$ of patients who presented with apparent disease progression on the first post-RT magnetic resonance imaging (MRI) actually had radiographic pseudoprogression. Although no specific etiology is known, it is postulated that radiographic pseudoprogression is due to either a transient intense inflammatory response to therapy ${ }^{3,4}$ or treatment-induced tumor cell death and necrosis ${ }^{5,7}$. Brandes and colleagues ${ }^{7}$ hypothesized that this could represent glioma killing effects of the treatment since they found a significant association with better outcomes in patients with pseudoprogression in comparison to those with stable MRI or with real progression. Most of the patients with pseudoprogression in their study harbored a tumor with methylated MGMT promoter. In their multivariate analysis survival was influenced not only by the MGMT status but also by the detection of pseudoprogression.

Radiographic pseudoprogression can be defined as an increase in gadolinium enhancement on T1-weighted images obtained post-concurrent therapy (MRIcon) which either stabilizes or improves radiographically after treatment with adjuvant TMZ. The Canadian GBM Recommendations Committee recently recommended that patients with evidence of radiological progression who have stable clinical symptoms during concurrent treatment should complete three cycles of adjuvant therapy before a decision is made about whether to discontinue treatment ${ }^{8}$. This recommendation is based on the supposition that in the first few weeks or months following completion of radiotherapy, MRI changes alone are not a reliable indicator of true progression.

We chose to examine the accuracy of this assertion by conducting a retrospective population-based analysis to evaluate the incidence of radiographic pseudoprogression in GBM patients after concurrent treatment and to determine its relationship to patient outcome and MGMT promoter methylation status. Furthermore, we endeavored to confirm the observation of Brandes et $\mathrm{al}^{7}$ who demonstrated that determination of MGMT promoter status could contribute to the treatment planning in patients who develop MRI progression early after concurrent treatment.

The relevance of this topic in the clinical practice of Neurooncology is reflected by the fact that this paper was reported at the 2008 Canadian Neuro-Oncology meeting and its abstract was published in the Canadian Journal of Neurological Sciences.

\section{Materials And Methods}

\section{Inclusion and Exclusion Criteria}

Ethical approval was received from the Conjoint Health Research Ethics Board of the University of Calgary to review charts and imaging data for every patient diagnosed with GBM (WHO grade IV glioma) who received chemoradiotherapy in southern Alberta, Canada from January 2004 to December 2006. The catchment area for southern Alberta is approximately two million people. Inclusion criteria were as follows: age 18 years or older, pathologically confirmed GBM, an initial postoperative MRI obtained less than 72 hours after surgical resection (baseline MRI), and treatment with concurrent radiation and low dose temozolomide (60 Gy in 6-7 weeks and concurrent 75 $\mathrm{mg} / \mathrm{m}^{2}$ of temozolomide daily for 42-49 days). Patients were required to have had an MRI scan 4-6 \pm 1 week post-concurrent treatment. Only patients on stable doses of steroids at least seven days before the MRI were included in the analysis. These imaging criteria comply with those described by Stupp et $\mathrm{al}^{1}$. Patients receiving less than $75 \%$ of the planned dose of radiation ( $<45$ Gy) or chemotherapy ( $<30$ daily doses) during the concurrent phase of therapy were excluded. Charts were reviewed for age, Karnofsky Performance Score (KPS), date and extent of surgery, date of start and finish of concurrent treatment, total doses, tolerance, admissions, and dexamethasone dosing during and after treatment until the post-concurrent treatment scan was obtained. All chart analysis was done by one person who was blinded to the radiographic and molecular results.

\section{MRI Analysis}

Magnetic resonance imaging studies were evaluated by a single neuro-radiologist who was blinded to clinical and

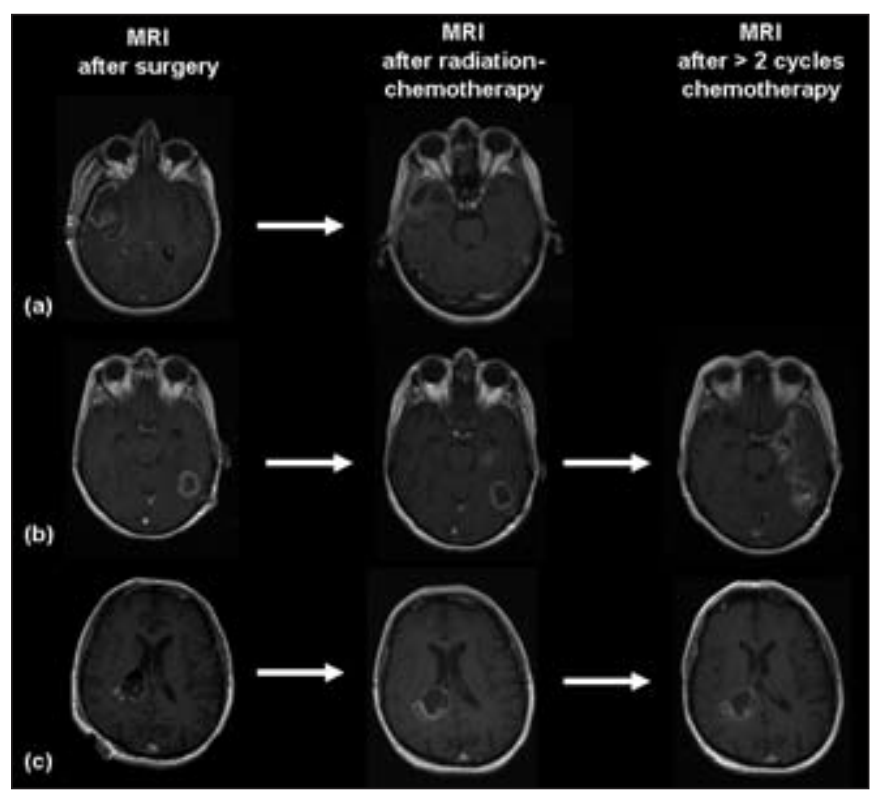

Figure: T1 sequence with gadolinium images of patients with GBM. (a) MRIcon showed partial response. (b) MRIcon shows progression of the disease that is confirmed in MRIadj. (c) MRIcon shows worsening of the disease that is proved as pseudoprogression in the MRI adj. 


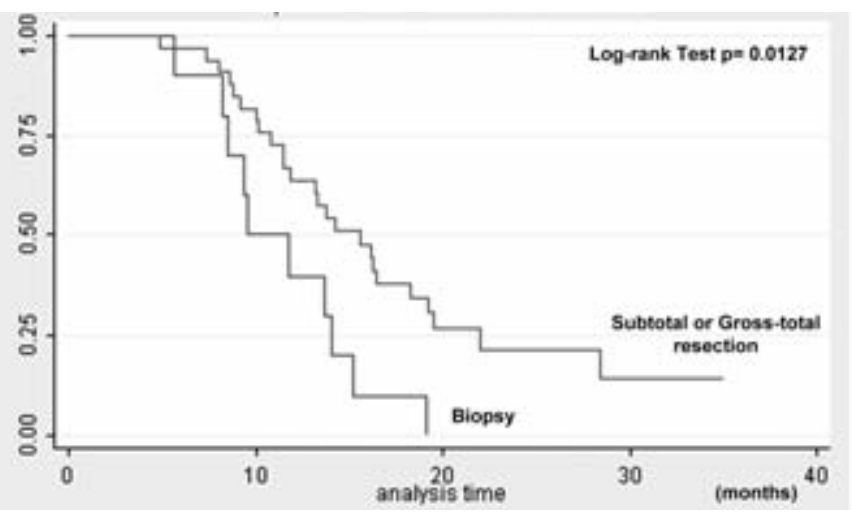

Figure 2: Overall survival of 43 GBM patients who completed concurrent treatment with low dose temozolomide and radiation according to extent of the initial resection.

molecular characteristics. The baseline postoperative MRI (taken less than $72 \mathrm{hrs}$ after surgery) was compared to the scan obtained at completion of concurrent treatment (MRIcon). If radiographic progression was seen on the MRIcon, a further comparison was made with the MRI performed after 2-3 cycles of adjuvant TMZ (MRIadj). The evaluation focused on T1 enhanced images to define tumor progression as according to the Macdonald criteria in which progressive disease represents a $25 \%$ or greater increase in largest cross sectional tumor area or appearance of a new lesion?.

\section{True Radiographic Progression Versus Radiographic Pseudoprogression}

True radiographic progression was defined as radiographic progression on the MRIcon which deteriorated further on the

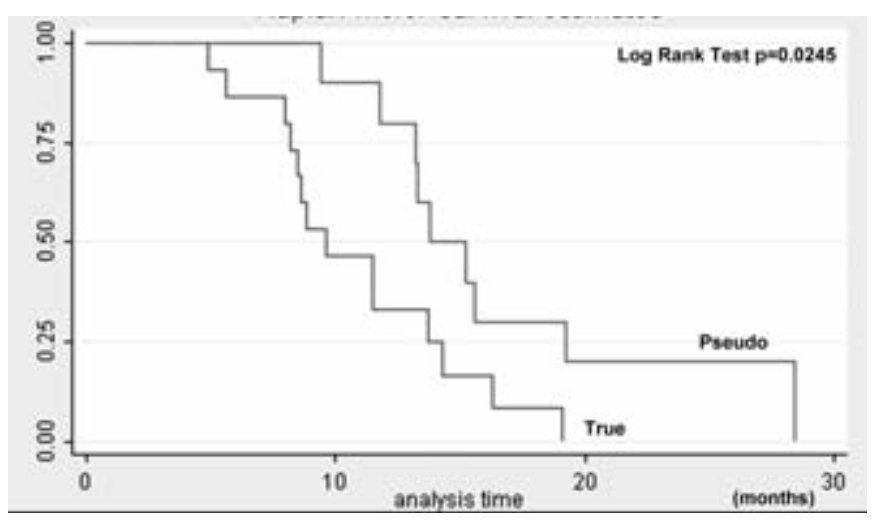

Figure 4: Overall survival of 20 GBM patients receiving adjuvant temozolomide in addition to concurrent temozolomide-radiation according to evidence of either true progression (True) or pseudoprogression (Pseudo).

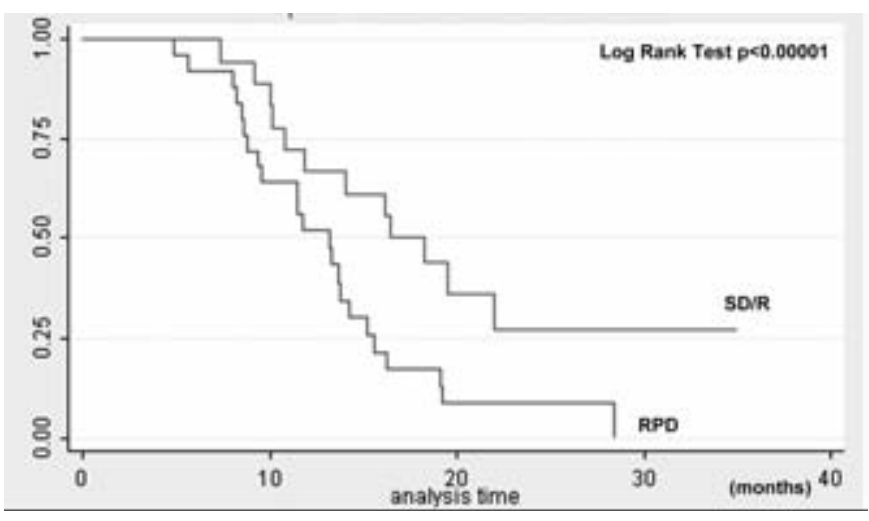

Figure 3: Overall survival of 43 GBM patients treated with concurrent temozolomide-radiation according to the first radiographic evaluation acquired after completion of concurrent therapy. $R P D=$ radiographic progressive disease; $S D / R=$ Stable disease or response .

MRIadj (done after receiving at least one cycle of adjuvant TMZ) (Figure 1). Radiographic pseudoprogression was defined as radiographic progression (i.e. increased $\mathrm{T} 1$ enhancement) on the MRIcon which either stabilized or resolved on the MRIadj after receiving adjuvant TMZ (Figure 1b).

\section{MGMT Promoter Methylation Status}

MGMT promoter methylation status was assessed by methylation-specific polymerase chain reaction (MS-PCR). One $\mu \mathrm{g}$ of DNA was subjected to bisulphite conversion according to the manufacturer's protocol (EZ DNA Methylation-Gold Kit, Zymo Research). Methylation-specific polymerase chain reaction was performed in two steps, as described elsewhere ${ }^{2}$. Analysis of the PCR gels was performed blinded to clinical and radiographic data.

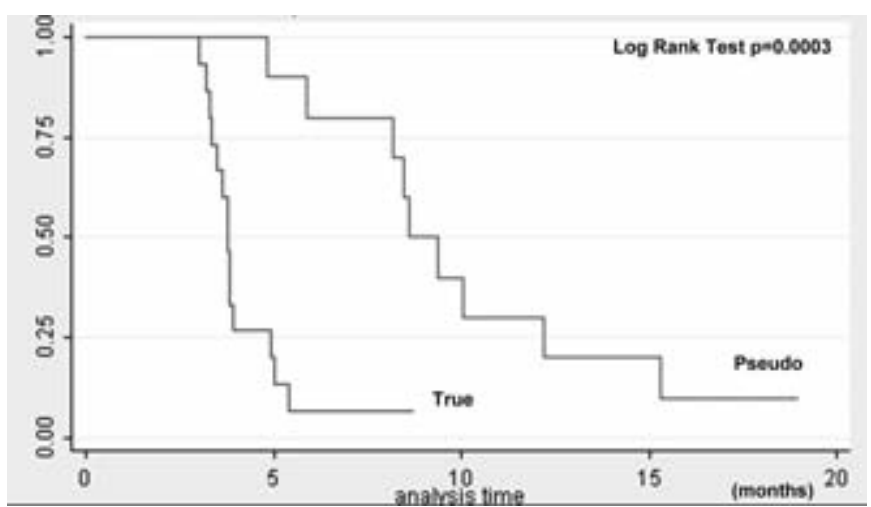

Figure 5: Progression-free survival of 20 GBM patients receiving adjuvant temozolomide in addition to concurrent temozolomideradiation according to evidence of either true progression (True) or pseudoprogression (Pseudo). 


\section{Statistics}

A comparison of patient characteristics between patient groups was carried out using Fisher's test for categorical variables and the Student's t test for continuous variables. The Kaplan-Meier method was used to estimate distributions for median survival (MS) and progression-free survival (PFS). The log-rank test was used to assess differences between these distributions with respect to the occurrence of pseudoprogression. All $\mathrm{p}$ values are two-sided and values less than 0.05 were considered significant. All calculations were done using STATA/IC version 10.0 software from StataCorp LP. PFS was calculated from the time of the surgery until evidence of progression in MRI (Macdonald criteria) ${ }^{9}$ or death from any cause. Median survival was defined as the time interval between the surgery and death or date of last visit.

\section{RESULTS}

A total of 142 patients were surgically treated and diagnosed with GBM between January 2004 and December 2006 in southern Alberta. Seventy-two of these patients were either unable (due to poor performance status, advanced age) or unwilling to undergo chemoradiotherapy. Of the remaining 70 patients who initiated postoperative concurrent treatment, 27 patients did not meet inclusion criteria: 3 did not complete concurrent treatment, 7 lacked a post-concurrent MRI and 17 patients were excluded because the interval between the end of the radiation and the MRI was less than three weeks (3 cases) or more than seven weeks (14 cases). We were very strict in our inclusion criteria in order to avoid including patients with confounding factors that would be wrongly categorized as pseudoprogressors. It was of particular relevance not to include MRIs taken too early or too late after concurrent treatment and patients that had modified steroid doses near this study was done. As a result, a total of 43 patients were included in this study.

Of these 43 patients, $25(58 \%)$ were male and the median age and KPS for all patients at diagnosis was 55 years (range $29-$ 71 ) and 80 (range $60-100$ ), respectively. The extent of the initial resection was gross total (>95\% of the initial enhancing tumor) in 10 cases (23\%), and subtotal in 23 cases (53\%). The remaining 10 patients had a diagnostic biopsy (23\%).

Postoperative concurrent treatment was initiated a median of 29 days from surgery (range 18-40 days). All patients included in the study received 60 Gy (median; range 54-60) in 6.1 weeks (range 4.7-7.9) with 42 doses (median; range 32-49) of daily low dose temozolomide.

The MRI scans were obtained at a median of 30 days (range 22-48) after completion of concurrent treatment, with 25 (58\%) patients experiencing progression, $13(30 \%)$ with stable disease, and $5(12 \%)$ showing partial responses. In all the cases the patients with progression had increased size of pre-existing lesions and none had new enhancing lesions.

Forty two patients (98\%) were taking $6 \mathrm{mg} / \mathrm{day}$ of dexamethasone (median; range 0-24) at the beginning of radiotherapy and $6 \mathrm{mg} /$ day (median; range $0.25-24$ ) at the end. During concurrent treatment 10 patients (24\%) remained on stable doses of dexamethasone, 18 (43\%) were able to decrease their dose, and $13(31 \%)$ required an increase in dexamethasone. There was no difference in dexamethasone dosing between patients who had a surgical biopsy versus those with a subtotal or gross total resection $(\mathrm{p}=1.0)$.

\section{Radiographic pseudoprogression}

Of the 25 patients with radiographic progression on MRIcon, $5(20 \%)$ were unable to receive adjuvant TMZ because of marked clinical deterioration or intolerance. The remaining 20 patients (Table) received a median of 2 (range 1-6) cycles of monthly temozolomide. In ten of these patients, further radiologic progression of the disease was observed in MRI scans acquired after 1 or 2 cycles implying that $50 \%$ of patients with radiographic progression on the MRIcon in the absence of clinical deterioration actually had true radiographic progression of disease. The remaining ten patients demonstrated radiographic pseudoprogression and benefited from adjuvant temozolomide by showing stable disease or response effect on the MRIadj.

Table: Characteristics of 20 GBM patients diagnosed with true radiographic progression versus radiographic pseudoprogression

\begin{tabular}{c|c|c|c|c|c|c}
\hline & $\begin{array}{c}\text { MS in months } \\
\text { (range) }\end{array}$ & $\begin{array}{c}\text { PFS in } \\
\text { months } \\
\text { (range) }\end{array}$ & Biopsy & $\begin{array}{c}\text { Median } \\
\text { age in } \\
\text { years }\end{array}$ & $\begin{array}{c}\text { Median KPS } \\
\text { (range) }\end{array}$ & $\begin{array}{c}\text { Tumors with methylated MGMT I } \\
\text { tumors successfully tested }\end{array}$ \\
\hline $\begin{array}{c}\text { True radiographic } \\
\text { progression (n=10) }\end{array}$ & $9.1(4.9-19.1)$ & $3.8(3.2-5)$ & $5 / 10$ & 59.5 & $80(60-90)$ & $3 / 6$ \\
\hline $\begin{array}{c}\text { Radiographic } \\
\text { pseudoprogression } \\
(\mathrm{n}=10)\end{array}$ & $14.5(9.4-28.4)$ & $9(4.8-15.3)$ & $3 / 10$ & 51 & $80(70-100)$ & $6 / 9$ \\
\hline
\end{tabular}

MS: median survival; PFS: median progression free survival; KPS: Karnofsky Performance Score. 


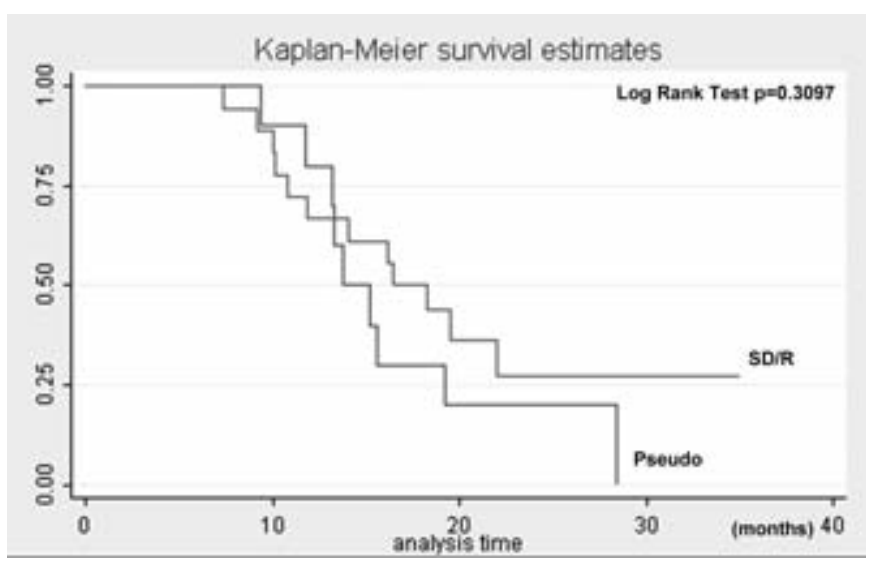

Figure 6: Overall survival of GBM patients who had radiographic response or stable disease $(S D / R)$ in the first MRI acquired after concurrent treatment versus those who had pseudoprogression (Pseudo).

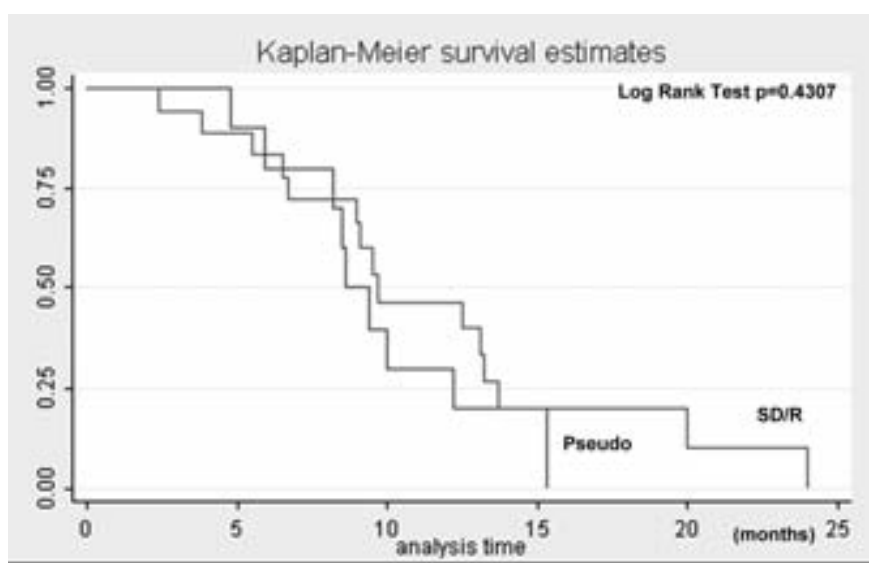

Figure 7: Progression-free survival of GBM patients who had radiographic response or stable disease $(S D / R)$ in the first MRI acquired after concurrent treatment versus those who had pseudoprogression (Pseudo).

9.1 months (range 4.9-19.1) and PFS of 3.8 months (range 3.25) while patients with radiographic pseudoprogression $(n=10)$ lived a median of 14.5 months (range 9.4-28.4; $\mathrm{p}=0.0245$ ) and progressed by 9 months (range 4.8-15.3; p=0.0003) (Figures $4,5)$.

No difference in either MS $(p=0.3097)$ or PFS $(p=0.4307)$ was seen between patients who responded/stabilized after concurrent treatment and those who had radiographic pseudoprogression (Figure 6,7).

\section{DisCUSSION}

The addition of TMZ to radiotherapy represents an important advance in the treatment of patients with GBM $^{1}$. Increasingly, clinicians are reporting that patients with radiologic progression on MRIcon experience radiologic stabilization or improvement after treatment with adjuvant temozolomide. We designed this retrospective study to evaluate the incidence and clinical significance of radiographic pseudoprogression in southern Alberta. The fact that we were unable to include all the patients constitutes a limitation of every retrospective analysis and we found some heterogeneity in therapy as commonly observed in population-based studies.

In our study, 20 patients received at least one cycle of adjuvant temozolomide after progression was seen on MRIcon. In $50 \%$ of these cases, the disease stabilized or improved and was consistent with radiographic pseudoprogression, while the rest radiographically progressed (i.e. true radiographic progression). This data corroborates the study published by Taal et $\mathrm{al}^{5}$, who also described a $50 \%$ incidence in radiographic pseudoprogression in GBM patients in a single institution study. The PFS and MS of patients with radiographic pseudoprogression were statistically identical to patients with no evidence of progression on MRIcon, suggesting that the etiology of radiographic pseudoprogression is likely benign, in agreement with earlier reports ${ }^{3-5}$. 
MGMT promoter methylation status in GBM tumors has been identified as a prognostic and predictive factor for patient survival and response to therapy, respectively ${ }^{2}$. We initially hypothesized that the phenomenon of pseudoprogression would be restricted to patients with treatment-sensitive MGMT methylated tumors and that pseudoprogression could be distinguished from true progression by knowing the MGMT status at diagnosis. However, our sample size was too small and the statistical power was too low to draw definitive conclusions regarding the association between MGMT promoter methylation and the occurrence of pseudoprogression. This hypothesis, however, appears to be supported by a recent study by Brandes et $\mathrm{al}^{7}$ who observed MGMT promoter methylation in $65 \%$ $(21 / 32)$ of the patients with pseudoprogression and in only $11 \%$ $(2 / 18)$ of the real progressors. Interestingly, Brandes reported a lower incidence of progression in the first scan $(48.5 \%)$ versus our study $(58 \%)$, however, they observed pseudoprogression more frequently $(58 \%)$ than we did $(50 \%)$. These discrepancies may be explained by differences in the inclusion/exclusion criteria between the two studies. We excluded patients based on changes in steroids dosage near the dates of MRI scans whereas Brandes did not. Since it is known that changes in steroid dosing can alter the appearance of MRI scans, we cannot be sure that the population assessed in Brandes' study is homogeneous. Also, we strictly excluded any patient who did not have an MRIcon four to six weeks after the completion of concurrent therapy whereas a similar date range for MRIcon was not explicitly stated in Brandes' report.

To our knowledge, this is the first population-based study evaluating the incidence of pseudoprogression in GBM patients treated with concurrent RT and TMZ. Our results suggest that adjuvant therapy should still be given to patients with progression seen on MRIcon as nearly half of these patients will stabilize or improve with further therapy. These findings are in agreement with the Canadian GBM Recommendations Committee, which recommends that patients with GBM should be treated for at least three additional cycles of chemotherapy if progression is seen on the MRI done shortly after the completion of $\mathrm{RT}^{8}$. Finally, these data may bring into question the clinical utility of the practice of performing an MRI immediately following concurrent therapy in stable patients because the results of an MRI scan alone at this time cannot be used to determine whether therapy should be stopped or continued. However, taking into account the existence of pseudoprogression, it would be needed to identify this group of patients with differential outcome. This hypothesis may be best tested in a prospective trial.

\section{REFERENCES}

1. Stupp R, Mason WP, van den Bent MJ, Weller M, Fisher B, Taphoorn MJ, et al. Radiotherapy plus concomitant and adjuvant Temozolomide for Glioblastoma. N Engl J Med. 2005;352: 987-96.

2. Hegi ME, Diserens AC, Gorlia T, Hamou MF, de Tribolet N, Weller $\mathrm{M}$, et al. MGMT gene silencing and benefit from temozolomide in glioblastoma. N Engl J Med. 2005;352:997-1003.

3. Rosenthal M, Ashley D, Cher L. Temozolomide-induced flare in high-grade gliomas: a new clinical entity. Intern Med J. 2002; 32:346-8.

4. De Wit M, de Bruin H, Eijkenboom W, Sillevis P, van den Bent M. Immediate post-radiotherapy changes in malignant glioma can mimic tumor progression. Neurology. 2004;63:535-7.
5. Taal W, Brandsma D, de Bruin H, Bromberg J, Swaak-Kragten A, Sillevis Smith P, et al. Incidence of early Pseudo-progression in a cohort of malignant glioma patients treated with chemoirradiation with temozolomide. Cancer. 2008;113:405-10.

6. Chamberlain M, Glantz M, Chalmers L, Van Horn A, Sloan A. Early necrosis following concurrent Temodar and radiotherapy in patients with glioblastoma. J Neurooncol. 2007; 82:81-3.

7. Brandes A, Franceschi E, Tosoni A, Blatt V, Pession A, Tallini G, et al. MGMT promoter methylation status cvan predict the incidence and outcome of pseudoprogression after concomitant radiochemotherapy in newly diagnosed glioblastoma patients. J Clin Oncol. 2008;26:2192-7.

8. Mason W, Del Maestro R, Eisenstat D, Forsyth P, Fulton D, Laperriere $\mathrm{N}$, et al. Canadian Recommendations for the treatment of glioblastoma multiforme. Curr Oncol. 2007; 14 (3): 110-7

9. Macdonald D, Cascino T, Schold C, Cairncross G. Response criteria for Phase II studies of supratentorial malignant glioma. J Clin Oncol. 1990;8:1277-80. 